\title{
Dietary Diversity in Relation to Micronutrient Adequacy in the Diets of Adolescents of Fatehabad Haryana, India
}

\author{
Nisha Rani and Varsha Rani* \\ Department of Foods and Nutrition, COHS, CCS Haryana Agricultural University, \\ Hisar, Haryana, India \\ *Corresponding author
}

\section{A B S T R A C T}

Dietary diversity (DD) has been identified as a potentially useful indicator of micronutrient status. Adolescents eating less number of food groups may be at the risk of nutritional deficiency. This study was aimed to determine the dietary diversity in the diets of adolescents and to find its correlation with the probability of adequacy of five micronutrients. Two hundred adolescents (13-17y) were selected randomly from the rural Govt. Senior Secondary School of Dhanger and urban Govt. Senior Secondary School Fatehabad, Haryana. To reflect the dietary diversity, two variables were calculated for each individual: Dietary Diversity Scores (DDS) and Food Variety Scores (FVS). Total food items consumed on two non-consecutive days were categorized into 13 food groups. The DDS was then calculated as the sum of all food groups consumed by an individual. The total score could range from 0 to 13. Probabilities of adequacy (PA) of five micronutrients i.e. for vitamin A, vitamin C, calcium, iron and zinc was calculated based on the Estimated Average Requirement (EAR). Scores for DDS and FVS did not range to the highest possible score. Mean value of DDS was $6.46( \pm 1.05)$. The theoretical range was 1 to 13 , while in the sample it varied from 4 to 8 . The mean probability of adequacy across five micronutrients among rural adolescents was 57 per cent which was 49 per cent in the urban adolescents. The diets of boys and urban adolescents were more diversified though the limit was not reached to the possible maximum ranges. Adolescents should be educated and motivated to eat all group fruits and vegetables.

\section{Introduction}

Micronutrient deficiency is an alarming public health problem; it undermines the health, development, and academic potential of millions of children and adolescents globally (FAO, 2002). All around the world about $60-80$ per cent of the adolescents have been facing micronutrient deficiency. In India alone more than 50 per cent of adolescent girls and 30 per cent of adolescent boys are iron deficient; the situation is worst in some states like Uttar Pradesh, Bihar, Rajasthan,
Orissa, Jharkhand and many more. Adolescent girls in Chennai and Tamil Nadu were found to be affected by severe $(6 \%)$, moderate $(35 \%)$ and mild $(38 \%)$ type of anemia (Premalatha et al., 2012). Micronutrients play a crucial role in the development and maintenance of good health. Adolescents, at the peak of their growth velocity require a large quantity of micronutrients. The increment in skeletal mass, body size and body density, associated 
with pubescence, highlights the role of micro nutrients along with the macro nutrients in the growth process. Irrespective of food availability, low bio-availability of nutrients, poor hygiene and drinking water, parasitic infections and faulty eating habits might be the constraints against low micronutrient status of adolescents in India. Micronutrient deficiency can have long term consequences such as impaired behavioral and brain development, depression, delayed sexual maturation and loss of final adult height.

Dietary diversity (DD), which is defined as the number of food groups consumed over a reference period has been identified as a potentially useful indicator of micronutrient status (Arsenault et al., 2013; Nguyen et al., 2013). Along with micronutrient status it is also correlated with macronutrient intakes as well as various anthropometric measures; measurements are simple to collect and easily adapted to diet in various settings. They have been used to study diet in both early childhood and adulthood (Daniels et al., 2009). The multiple micro nutrients intake is reflected through probability of adequacy. At individual level, dietary diversity is usually reflects the dietary quality (FAO, 2011). Dietary diversity appliance has recently become the preferred method for studying dietary adequacy in developing country like India. In a study, association between dietary diversity and micronutrient adequacy was assessed in the diets of women in reproductive age in five countries and found that dietary diversity was significantly associated with micronutrient adequacy in all sites (Arimond et al., 2010). The nutrient density in the diet of adolescents is often inadequate to achieve increased nutritional requirement and therefore escalating the diversity of foods provided such as fruits and vegetables, egg, meat, poultry and fish is recommended to improve micronutrient intakes.
Probability of adequacy (PA) is the likelihood that an individual's intake of one particular nutrient is adequate over the given requirement distribution. The estimated average requirements (EAR) and the standard deviation (SD) are used to calculate PA. PA takes the distribution of the nutrient intake of the population into account and the proportion of individuals at risk for inadequate intakes can be estimated. DD and PA are strongly associated with socio economic factors such as family income, family education and family occupation. As income increases, the possibility that people diversify their diets also increases. This is due to the fact that varied diets generally are more palatable and more pleasant. Research has shown the association between dietary diversity and SES and food security.

A few studies have scrutinized the contribution of individual food groups to nutrient adequacy but the role of dietary patterns has been rarely examined in either children or adolescents (Welch et al., 2009; Gao et al., 2006). One such study of young adults demonstrated that those who ate adequate food groups were more likely to have adequate intakes of several important nutrients (Song et al., 1996). This study was focused on various food group intakes among adolescents of rural and urban backgrounds and to explore the correlation between dietary diversity and probability of adequacy of micronutrients.

\section{Materials and Methods}

\section{Sample selection}

This study was carried out in Fatehabad district of Haryana State. Two hundred school going adolescents (13-17y) were selected randomly from the rural Govt. Senior Secondary School of Dhanger village, Fatehabad and urban Govt. Senior Secondary 
School of Fatehabad city. The proportion of male and female students was assigned equal while selecting the respondents.

\section{Dietary diversity}

To reflect the dietary diversity, two variables were calculated for each individual: Dietary Diversity Scores (DDS) and Food Variety Scores (FVS). Thirteen food groups were created following the methodology used in another study (Arimond et al., 2007). The information on dietary intake among adolescents was collected using $24 \mathrm{~h}$ recall method.

\section{Food groups}

The food groups created for calculating dietary diversity score in this study were: 1 . All starchy staples; 2. All legumes and nuts; 3. All Dairy; 4. Organ meat; 5. Eggs; 6. Small fish eaten whole with bones; 7. All other flesh foods and miscellaneous, small animal protein; 8. Vitamin A-rich dark green leafy vegetables; 9. Vitamin A-rich deep yellow, orange and red vegetables; 10. Vitamin A-rich fruits; 11. Vitamin C-rich vegetables; 12. Vitamin C-rich fruits; and 13. All other fruits and vegetables. Other food items like fats and oils, sugars, and other beverages were not taken into account because they only contribute to energy intake and not to micronutrient intake.

\section{Dietary diversity scores and food variety scores}

When food items within a food group were consumed by a subject during the two recall days, this food group received a score of one point for DDS. When no food items within a food group were eaten a score of 0 was given. DDS increases with length of recall period. The DDS was then calculated as the sum of all food groups consumed by an individual.
The total score could range from 0 to 13 . To calculate FVS, all food items consumed by the subjects during the two recall days were counted and summed. The food items that were categorized in the 13 food groups were taken into account for this calculation. The total score of FVS was ranged from 0 to 83 in present study.

\section{Nutrient adequacy}

Probabilities of adequacy (PA) of five micronutrients i.e. for vitamin $\mathrm{A}$, vitamin $\mathrm{C}$, calcium, iron and zinc was calculated based on the Estimated Average Requirement (EAR). PA was calculated by the equation $\mathrm{PA}=[$ (observed individual intake EAR)/Standard Deviation] using function CDFNORM, where CDFNORM is the statistical function from SPSS that calculates the probability. Mean probability of adequacy (MPA) was calculated as the sum of probabilities of 5 micronutrients.

\section{Statistical analysis}

Statistically data was analyzed using SPSS statistical package (version 14.0) for windows. Bivariate (Pearson) correlations were conducted to explore the relationship between DDS and FVS and probability of adequacy of micronutrients (adjusted for energy intake, age, sex and socio economic status (SES) proxies).

\section{Results and Discussion}

\section{Dietary diversity score and food variety score}

Scores for DDS and FVS did not range to the highest possible score. Mean value of DDS was $6.46( \pm 1.05)$. The theoretical range was 1 to 13 , while in the sample it varied from 4 to 8. Eighty one different food items were reported to be eaten by the adolescents during 
the survey period. Mean FVS was $16.45( \pm$ 2.85 ), with a minimum intake of 6 and a maximum of 24. Area-wise comparison showed that urban adolescents had significantly $(p<0.01))$ higher scores of dietary diversity and food variety than rural adolescents (Table 1). The dietary diversity score and food variety score of urban adolescents was 6.92 and 17.36, respectively while the diversity score and food variety score of rural adolescents was 6.01 and 15.68, respectively. Gender-wise comparison showed that boys had more scores of dietary diversity and food variety than girls however a non significant difference for dietary diversity score but significant difference for food variety score was observed (Table 1). The dietary diversity score and food variety score of boys was 6.57 and 17.29, respectively while the diversity score and food variety score of girls was 6.36 and 15.60, respectively.

\section{Probability of adequacy of micronutrients}

In general, all the estimates of probability of adequacy for micronutrients ranged low. Intakes of iron had the lowest $(30 \%)$ while intakes of calcium had the highest $(70 \%)$ probability of adequacy followed by the vitamin A (61\%), zinc (54\%) and vitamin C $(51 \%)$. The mean probability of adequacy across 5 micronutrients was 53 percent (Table 2).

The mean probability of adequacy across five micronutrients among rural adolescents was 57 per cent which was 49 per cent in the urban adolescents. Area-wise comparison of probability of adequacy among adolescents indicated that rural adolescents had the significantly $(p<0.01)$ higher probability of adequacy of vitamin $A$ whereas the probability of adequacy of iron was significantly $(p<0.01)$ higher in urban adolescents (Table 2). The mean probability of adequacy across five micronutrients of boys was 60 per cent which was 46 percent in girls. Gender-wise comparison of probability of adequacy among adolescents indicated that boys had the significantly $(p<0.01)$ higher probability of adequacy of calcium, zinc and mean probability of adequacy across 5 micronutrients (Table 2).

Pearson's correlations coefficients of dietary diversity scores and adequacy of micronutrient were significant for probability of adequacy of iron, vitamin $\mathrm{C}$, vitamin $\mathrm{A}$, and mean probability of adequacy $(\mathrm{r}=0.38$, $\mathrm{r}=0.25, \quad \mathrm{r}=0.28$ and $\mathrm{r}=0.28$ respectively; $p<0.01)$. The association of iron, vitamin $\mathrm{C}$ and mean probability of adequacy were increased after adjusting for income and association of vitamin A were decreased after adjusting for protein intake. The rest associations remained unchanged but significant after adjusting for protein intake, energy intake, age, gender and income (Table $3)$.

Pearson's correlations coefficients of food variety scores and adequacy of micronutrient were significant for probability of adequacy of calcium, iron, vitamin $\mathrm{C}$, zinc, vitamin $\mathrm{A}$, and mean probability of adequacy $(r=0.19$, $\mathrm{r}=0.31, \quad \mathrm{r}=0.27, \quad \mathrm{r}=0.23, \quad \mathrm{r}=0.27$ and $\mathrm{r}=0.15$ respectively; $p<0.01)$. The association of iron and vitamin $\mathrm{A}$ were increased after adjusting for income and association of vitamin A were decreased after adjusting for protein intake. The rest associations remained unchanged but the effect was not significant after adjusting for energy intake, age, gender and income (Table 4).

Regarding the consumption of different food groups, none of the adolescents had consumed organ meat, small fish eaten whole with bones and all other flesh foods and miscellaneous small animal protein as majority of the respondents were vegetarian. 
Table.1 Area-wise and gender-wise comparison of $\mathrm{DDS}^{1}$ and $\mathrm{FVS}^{2}$

\begin{tabular}{|c|c|c|c|}
\hline & & $\begin{array}{c}\text { DDS }^{1} \\
\text { (13 sub food groups) }\end{array}$ & $\begin{array}{c}\text { FVS }^{2} \\
\text { (83 sub food items) }\end{array}$ \\
\hline \multicolumn{2}{|c|}{ Total (200) } & $6.46 \pm 1.05$ & $16.45 \pm 2.85$ \\
\hline \multirow[t]{3}{*}{ Area-wise } & Rural (100) & $6.01 \pm 1.03$ & $15.68 \pm 2.36$ \\
\hline & Urban (100) & $6.92 \pm 0.86$ & $17.36 \pm 2.59$ \\
\hline & t-value & $3.74^{* *}$ & $4.79^{* *}$ \\
\hline \multirow[t]{3}{*}{ Gender-wise } & Boys (100) & $6.57 \pm 1.15$ & $17.29 \pm 2.72$ \\
\hline & Girls (100) & $6.36 \pm 0.93$ & $15.60 \pm 2.74$ \\
\hline & t-value & $1.41^{\mathrm{NS}}$ & $4.35^{* *}$ \\
\hline
\end{tabular}

Values are mean $\pm \mathrm{SD} ;{ }^{1}$ Dietary diversity score: $\min : 4$, max: $8 ;{ }^{* *}$ Significant at $1 \%$ level; ${ }^{2}$ Food variety score: $\min$ : 6, max: 24

Table.2 Area-wise comparison of probability of adequacy of micronutrients among adolescents

\begin{tabular}{|l|c|c|c|c|}
\hline Micronutrients & Total (200) & Rural (100) & Urban (100) & t-value \\
\hline Calcium & $0.70 \pm 0.52$ & $0.79 \pm 0.54$ & $0.60 \pm 0.51$ & $1.04^{\mathrm{NS}}$ \\
\hline Iron & $0.30 \pm 0.24$ & $0.22 \pm 0.19$ & $0.38 \pm 0.26$ & $3.46^{* *}$ \\
\hline Vitamin C & $0.51 \pm 0.26$ & $0.48 \pm 0.25$ & $0.53 \pm 0.27$ & $0.18^{\mathrm{NS}}$ \\
\hline Zinc & $0.54 \pm 0.24$ & $0.57 \pm 0.23$ & $0.50 \pm 0.26$ & $0.60^{\mathrm{NS}}$ \\
\hline Vitamin A & $0.61 \pm 2.88$ & $0.79 \pm 2.64$ & $0.44 \pm 3.10$ & $3.30^{* *}$ \\
\hline Mean & $0.53 \pm 0.34$ & $0.57 \pm 0.34$ & $0.49 \pm 0.46$ & $1.04^{\mathrm{NS}}$ \\
\hline
\end{tabular}

Mean micronutrient intake over 2 days

Values are mean SD

** Significant at $1 \%$ level

Table.3 Correlation between probability of adequacy of micronutrients and DDS ${ }^{1}$ (Controlled for protein intake, energy intake, sex, age and income)

\begin{tabular}{|c|c|c|c|c|c|c|}
\hline \multirow{2}{*}{ Micronutrients } & \multicolumn{7}{|c|}{ DDS } \\
\cline { 2 - 7 } & Uncontrolled & $\begin{array}{c}\text { Controlled } \\
\text { for protein } \\
\text { intake }\end{array}$ & $\begin{array}{c}\text { Controlled } \\
\text { for energy } \\
\text { intake }\end{array}$ & $\begin{array}{l}\text { Controlled } \\
\text { for gender }\end{array}$ & $\begin{array}{c}\text { Controlled } \\
\text { for age }\end{array}$ & $\begin{array}{c}\text { Controlled } \\
\text { for income }\end{array}$ \\
\hline Calcium & 0.17 & 0.05 & 0.07 & 0.07 & 0.07 & 0.05 \\
\hline Iron & $0.38^{* *}$ & $0.38^{* *}$ & $0.38^{* *}$ & $0.38^{* *}$ & $0.38^{* *}$ & $0.42^{* * *}$ \\
\hline Vitamin C & $0.25^{* *}$ & $0.25^{* *}$ & $0.25^{* *}$ & $0.25^{* *}$ & $0.25^{* *}$ & $0.28^{* *}$ \\
\hline Zinc & 0.09 & 0.07 & 0.09 & 0.09 & 0.09 & 0.09 \\
\hline Vitamin A & $0.28^{* *}$ & $0.25^{* *}$ & $0.28^{* *}$ & $0.28^{* *}$ & $0.28^{* *}$ & $0.28^{* *}$ \\
\hline Mean & $0.28^{* *}$ & $0.28^{* *}$ & $0.28^{* *}$ & $0.28^{* *}$ & $0.28^{* *}$ & $0.38^{* *}$ \\
\hline
\end{tabular}

Significant at 5\% level ${ }^{* *}$ Significant at $1 \%$ level

${ }^{1}$ Dietary diversity score 
Table.4 Correlation between probability of adequacy of micronutrients and $\mathrm{FVS}^{1}$ (Controlled for protein, energy, sex, age and income intake)

\begin{tabular}{|l|c|c|c|c|c|c|}
\hline \multirow{2}{*}{ Micronutrients } & \multicolumn{6}{|c|}{ FVS } \\
\cline { 2 - 7 } & Uncontrolled & $\begin{array}{c}\text { Controlled } \\
\text { for } \\
\text { protein }\end{array}$ & $\begin{array}{c}\text { Controlled } \\
\text { for energy }\end{array}$ & $\begin{array}{c}\text { Controlled } \\
\text { for sex }\end{array}$ & $\begin{array}{c}\text { Controlled } \\
\text { for age }\end{array}$ & $\begin{array}{c}\text { Controlled } \\
\text { for income }\end{array}$ \\
\hline Calcium & $0.19^{* *}$ & 0.11 & 0.13 & 0.00 & 0.01 & 0.05 \\
\hline Iron & $0.31^{* *}$ & $0.31^{* *}$ & 0.31 & 0.31 & 0.31 & $0.38^{* *}$ \\
\hline Vitamin C & $0.27^{* *}$ & 0.10 & 0.10 & 0.10 & 0.10 & 0.15 \\
\hline Zinc & $0.23^{* *}$ & 0.08 & 0.11 & 0.11 & 0.11 & 0.11 \\
\hline Vitamin A & $0.27^{* *}$ & $0.23^{* *}$ & 0.27 & 0.27 & 0.27 & $0.32^{*}$ \\
\hline Mean & 0.15 & 0.15 & 0.15 & 0.15 & 0.15 & 0.15 \\
\hline
\end{tabular}

Significant at $5 \%$ level ${ }^{* *}$ Significant at $1 \%$ level; ${ }^{1}$ Food variety score

Fig.1 Gender-wise comparison of probability of adequacy of micronutrients among adolescents

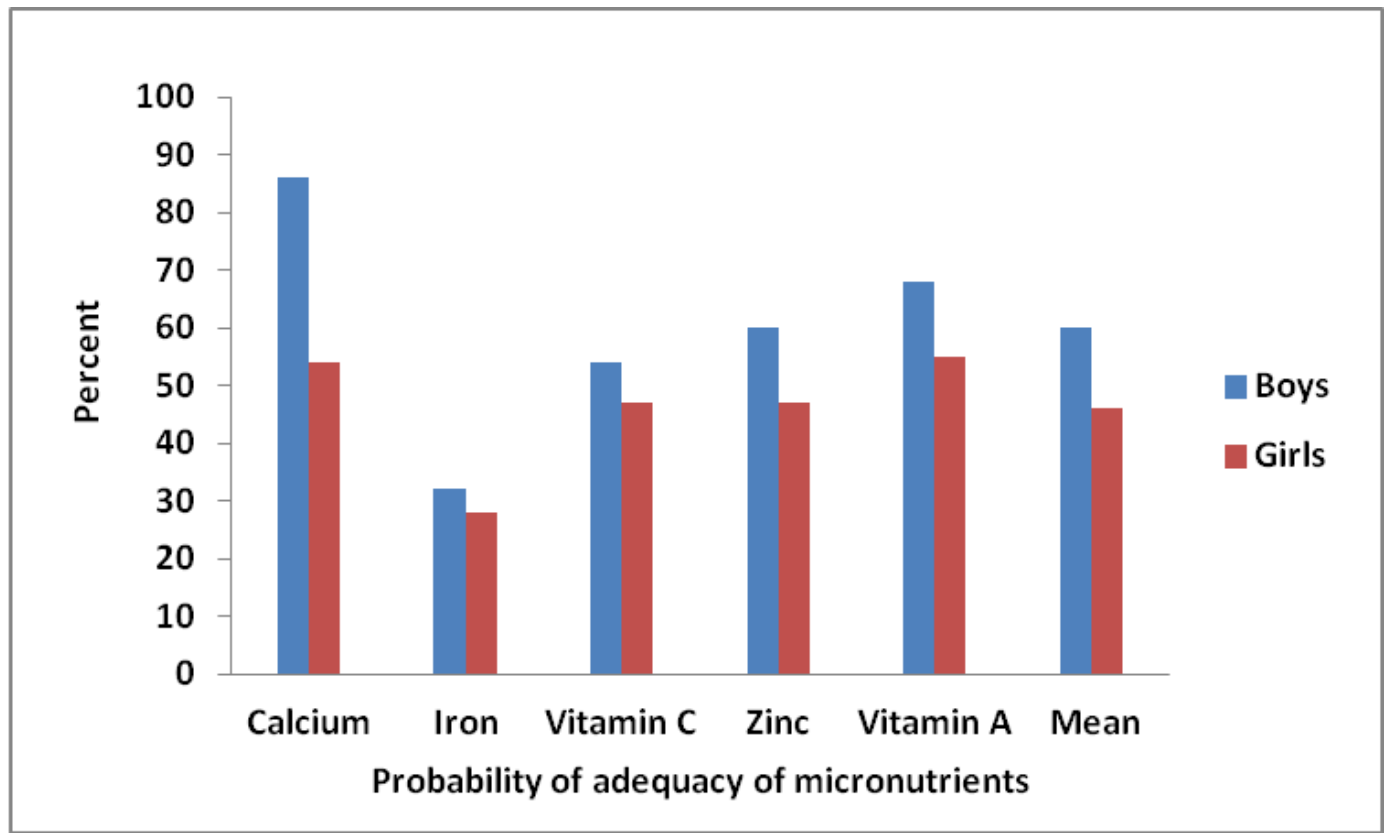

All of the adolescents consumed starchy staples and dairy products. All legumes and nuts were consumed by 94.5 per cent of the adolescents. Vitamin C-rich vegetables, $\beta$ carotene rich dark green leafy vegetables, all other fruits and vegetables, vitamin C-rich fruits, $\beta$-carotene rich deep yellow, orange and red vegetables, $\beta$-carotene rich fruits were taken by $60,57.5,54,33,16.5$ and 14.5 per cent of the students, respectively. Potts and
Potts (2014) observed that majority (48.23\%) of the preschool children consumed food items from 4 food groups. A smaller percent of the sample, 19.15 per cent, consumed $\leq 3$ food groups therefore indicating low dietary diversity. Only 6.15 per cent of the participants consumed a diversified diet from the six food groups; while 26 per cent consumed food items from five food groups. Fruits, vegetables and legumes were the food 
groups least consumed by the preschoolers. Food groups such as staples, dairy products and animal flesh foods were highly consumed by most of the participants. Highest number of food items consumed by this population was seventeen. Six to12 food items were consumed by most $(83.69 \%)$ of the children. A mean FVS of $9.37 \pm 2.45$ (range 2 to 17 ) was obtained. A cross-sectional survey was conducted among 232 children (5-8 years) of Hisar, Haryana using a $24 \mathrm{~h}$ recall. Food variety score (FVS) and Dietary diversity score (DDS) were calculated. Mean value of DDS was 6.5. Seventy-eight different food items were reported to be eaten by the children during the registration period. Mean FVS was 12.2. A cross-tabulation of DDS against the individual food groups was done to provide a picture of how diets diversify. Diets continue to diversify as scores increase (Rani et al., 2010).

In general, all the estimates of probability of adequacy for micronutrients ranged low. Intakes of iron had the lowest (30\%) while intakes of calcium had the highest $(70 \%)$ probability of adequacy followed by the vitamin A (61\%), zinc (54\%) and vitamin C $(51 \%)$. The mean probability of adequacy across 5 micronutrients was 53 percent (Table $2)$. The rural adolescents had the significantly $(p<0.01)$ higher probability of adequacy of vitamin A whereas the probability of adequacy of iron was significantly $(p<0.01)$ higher in urban adolescents. The boys had the significantly $(p<0.01)$ higher probability of adequacy of calcium, zinc and mean probability of adequacy across 5 micronutrients (Figure 1). The DDS and FVS were positively associated with probability of adequacy of micronutrients in present study. The contribution of food groups and subgroups to micronutrient adequacy depends on several factors and nutrient composition of the individual foods in a group or subgroup, the mix of foods eaten within the food group or subgroup, the amount of each consumed, and the correlation of intakes within and between food groups. For example, whole grain consumption might be correlated with fruit consumption among individuals focusing on a healthy diet while higher red meat intake might be correlated with consumption of starchy vegetables (e.g., French fries) in other individuals. Thus, correlated eating patterns may obscure the true nutrient contribution of individual foods or food groups. To address this issue, we constructed simple eating patterns that reflected the different ways in which foods may be eaten.

In our study, Pearson's correlations coefficients of dietary diversity scores and adequacy of micronutrient were significant for probability of adequacy of iron, vitamin $\mathrm{C}$, vitamin $A$, and mean probability of adequacy $(\mathrm{r}=0.38, \quad \mathrm{r}=0.25, \quad \mathrm{r}=0.28 \quad$ and $\mathrm{r}=0.28$ respectively; $p<0.01)$. The association of iron, vitamin $\mathrm{C}$ and mean probability of adequacy were increased after adjusting for income and association of vitamin A were decreased after adjusting for protein intake. The lack of association between DDS and the nutrient adequacy measures of calcium, vitamin $\mathrm{A}$, and zinc might be due to insufficient amounts consumed of foods containing these nutrients. FVS was significantly and stronger correlated with mean PA and mean MPA for all micronutrients, confirming other studies in different countries (Mali: $\mathrm{r}=0.33$ ) (Hatloy et al., 1998). However, a study done among Kenyan women found no association between FVS and nutrient adequacy measures (Diujzer, 2008). When the correlation coefficients of DDS and adequacy of micronutrient were controlled for protein intake, energy intake, age gender and income the association of iron, vitamin $\mathrm{C}$ and mean probability of adequacy were increased after adjusting for income and association of vitamin- A were decreased after adjusting for protein intake suggesting that income and 
protein intake might have some influence in this association. There was a significant relationship between the DDS and economic situation. Hatloy et al., (1998) found no correlation between FVS and DDS with adequacy of micronutrients in children aged 13-58 months. However, other studies did find a positive and significant association between food group diversity and micronutrient adequacy (Rani et al., 2010; Kennedy et al., 2010; Moore et al., 2012).

In this study a low dietary diversity was observed among adolescents. The diets of boys and urban adolescents were more diversified though the limit was not reached to the possible maximum ranges. Dietary diversity as the best indicator of probability of adequacy has been proven through this study since the probability of adequacy across five micronutrients was increased with the increasing dietary diversity in the diets of adolescents. Consumption of fruits and vegetable are important food groups in adding variety to the starchy diets therefore adolescents should be educated and motivated to eat all group fruits and vegetables.

\section{References}

Arimond, M., Wiesmann, D., Becquey, E., Carriquiry, A., Daniels, C. M., Deitchler, M., Torheim, L. E. 2010. Simple food group diversity indicators predict micronutrient adequacy of women's diets in 5 diverse, resourcepoor settings. J. Nutr., 140(11): 2059S2069S.

Arsenault, J.E., Yakes, E.A., Islam, M.M., Hossain, M.B., Ahmed, T., Hotz, C. 2013. Very low adequacy of micronutrient intakes by young children and women in rural Bangladesh is primarily explained by low food intake and limited diversity. J. Nutr., 143:197203.
Daniels, M.C., Adair, L.S., Popkin, B.M., Truong, Y.K. 2009. Dietary diversity scores can be improved through the use of portion requirements: an analysis in young Filipino children. Eur. J. Clin. Nutr, 63: 199-208.

Duijzer, G., 2008. The effect of using different food groupings on the association of dietary diversity and nutrient adequacy among women in Kenya, in Human Nutrition. Wageningen University: Wageningen. 53.

F.A.O., 2002. Human vitamin and mineral requirements. Food and Agriculture Organization of the United Nations, Rome, Italy.

F.A.O., 2011. Guidelines for measuring household and individual dietary diversity. Food and Agriculture Organization of the United Nations, Rome, Italy.

Gao, X., Wilde, P.E., Lichtenstein, A.H., Tucker, K.L. 2006. Meeting adequate intake for dietary calcium without dairy foods in adolescents aged 9 to 18 years (National Health and Nutrition Examination Survey 2001-2002). J. Am. Diet. Assoc., 106:1759-1765.

Hatloy, A., Torheim, L.E., Oshaug, A. 1998. Food variety - a good indicator of nutritional adequacy of the diet? A case study from an urban area in Mali, West Africa. Eur. J. Clin. Nutr, 52(12): 891898.

Kennedy, G., Fanou-Fogny, N., Chiara, N., Arimond, M., Koreissi, Y., Dossa, R., Frans J.K. and Brouwer, I.D. (2010) Food groups associated with a composite measure of probability of adequate intake of 11 micronutrients in the diets of women in urban Mali. $J$. Nutr., 140: 2070S-2078S.

Moore, L.L., Singer, M.R., Qureshi, M.M., Bradlee, M.L. Daniels, S.R. 2012. Food group intake and micronutrient 
adequacy in adolescent girls. Nutrient, 4: $1692-1708$.

Nguyen, P.H., Avula, R., Ruel, M.T., Saha, K.K., Ali, D., Tran, L.M. 2013. Maternal and child dietary diversity are associated in Bangladesh, Vietnam, and Ethiopia. J. Nutr., 143: 1176-1183.

Potts, S.C., Potts, A.C. 2014. An assessment of dietary diversity and nutritional status of preschool children. Austin. J. Nutr. Fd. Sci. 2(7): 1040.

Premalatha, T., Valarmathi, S., Srijayanth, P., Sundar, J.S. Kalpana, S. 2012. Prevalence of anemia and its associated factors among adolescent school girls in Chennai, Tamil Nadu, INDIA. Epidemiol. 2(2):118.

Rani, V., Arends E. D., Brouwer, I. 2010 Dietary diversity as an indicator of micronutrient adequacy of the diet of 5 to 8 year old Indian rural children. Nutr. Fd. Sci. 40(5): 466-476.

Song, W.O., Schuette, L.K., Huang, Y.L., Hoerr, S. 1996. Food group intake patterns in relation to nutritional adequacy of young adults. Nutr. Res., 16: 1507-1519.

Welch, A.A., Fransen, H., Jenab, M., Boutron-Ruault, M.C., Tumino, R., Agnoli, C., Ericson, U., Johansson, I., Ferrari, P., Engeset, D. 2009. Variation in intakes of calcium, phosphorus, magnesium, iron and potassium in 10 countries in the European Prospective Investigation into Cancer and Nutrition study. Eur. J. Clin. Nutr. 63 (4): S101S121.

\section{How to cite this article:}

Nisha Rani and Varsha Rani. 2017. Dietary Diversity in Relation to Micronutrient Adequacy in the Diets of Adolescents of Fatehabad Haryana. Int.J.Curr.Microbiol.App.Sci. 6(9): 302-310. doi: https://doi.org/10.20546/ijcmas.2017.609.038 